\title{
A Case of Sebaceous Carcinoma of the Submandibular Gland
}

\author{
Sung Hee Bae ${ }^{1}$, Min Jae Kim ${ }^{1}$, Sung Min Lee ${ }^{1}$, and Hae Sang Park ${ }^{1,2}$ \\ ${ }^{I}$ Department of Otorhinolaryngology-Head and Neck Surgery, Chuncheon Sacred Heart Hospital, College of Medicine, \\ Hallym University, Chuncheon; and ${ }^{2}$ Nano-Bio Regenerative Medical Institute, College of Medicine, Hallym University, \\ Chuncheon, Korea
}

\section{악하선에 발생한 지선암 1 예}

배승희 ${ }^{1} \cdot$ 김민재 $^{1} \cdot$ 이성민 ${ }^{1} \cdot$ 박혜상 $^{1,2}$

한림대학교 의과대학 춘천성심병원 이비인후-두경부외과학교실, ${ }^{1}$ 한림대학교 의과대학 나노 바이오 재생의학연구소 ${ }^{2}$

\author{
Received November 3, 2016 \\ Revised December 13, 2016 \\ Accepted December 15, 2016 \\ Address for correspondence \\ Hae Sang Park, MD, PhD \\ Department of Otorhinolaryngology- \\ Head and Neck Surgery, \\ Chuncheon Sacred Heart Hospital, \\ College of Medicine, \\ Hallym University, 77 Sakju-ro, \\ Chuncheon 24253, Korea \\ Tel +82-33-240-5181 \\ Fax +82-33-241-2909 \\ E-mail hs-piao@hanmail.net
}

Sebaceous carcinoma of the salivary gland is an extremely unordinary malignancy occurring mainly in the parotid gland. Sebaceous carcinoma arising in the submandibular gland is exceptional and only three cases have been reported in the literature. We herein present a unique case of sebaceous carcinoma of the submandibular gland, the first to be reported in literature in South Korea. An 85-year-old man visited our hospital complaining of a progressively enlarging submandibular mass with tenderness and ipsilateral facial palsy. We performed a wide excision of the mass with superficial parotidectomy and modified neck dissection. Microscopic and immunohistochemical examinations demonstrated sebaceous carcinoma of low grade differentiation. Further adjuvant radiotherapy was rejected and the patient was followed up for five months. The optimal treatment of sebaceous carcinoma of the salivary gland is still not established due to the rarity of the disease. When more cases are reported, the clinicopathologic characteristics will be better understood.

Korean J Otorhinolaryngol-Head Neck Surg 2018;61(5):258-62

Key Words Salivary gland neoplasms $\cdot$ Sebaceous carcinoma

Submandibular gland neoplasms.

\section{Introduction}

Based on the data, sebaceous glands can be occasionally found in normal salivary glands, $11 \%$ to $28 \%$ in the parotid glands, and $6 \%$ in the submandibular glands. ${ }^{1-3)}$ Malignant change of these glands, however, is extremely rare. In majority of these cases, sebaceous carcinoma occurs at the parotid gland and it is less commonly found in the submandibular and sublingual gland. ${ }^{4)}$ To date, 33 cases of sebaceous carcinoma of the parotid gland and only 3 cases involving the subman-

This is an Open Access article distributed under the terms of the Creative Commons Attribution Non-Commercial License (http://creativecommons.org/licenses/by-nc/4.0) which permits unrestricted non-commercial use, distribution, and reproduction in any medium, provided the original work is properly cited. dibular gland have been reported in the literature. ${ }^{4-7)}$

Most patients experience rapidly growing mass in the neck with or without pain, fixating to the overlying skin or deep muscle, ipsilateral facial palsy and cervical lymphadenopathy. ${ }^{89}$ Because of its rarity, there has been only few research on definite etiology, therefore, the relevant data on treatment is insufficient.

Due to its insufficiency of data, the gold standard treatment is still not fully established. As for current circumstances, wide surgical excision for low grade differentiation and adjuvant radiotherapy for high differentiations or positive margins are considered to be the most recommended treatment.

We encountered a huge mass at the submandibular gland 
which had been revealed to be sebaceous carcinoma, as the first one to be reported in literature in South Korea. The following report will discuss this case with a brief review on sebaceous carcinoma of the salivary glands.

\section{Case}

An 85-year-old Korean man visited our otolaryngology clinic with a chief complaint of left submandibular mass. The patient was aware of the mass since seven years ago but did not undergo any other diagnoses or treatments. In the early stages, the mass slowly progressed without tenderness. Two years ago, its size started to grow rapidly resulting in an ipsilateral facial palsy, then in a few months tenderness and erythematic change occurred. The patient had been diagnosed and also treated with hypertension and depression. He had a 15 pack-year of smoking history and had no significant fam- ily or social history.

Clinical examinations revealed a firm, fixed, and tender mass on the left submandibular area with a size of $10 \times 8 \mathrm{~cm}$. The mass border was irregular, spreading to the parotid tail area, and the overlying skin was erythematous with heat sensation. Left side facial palsy of a house-brackmann grade 4 was observed especially at the marginal area. Both nasal cavities were clear and further inspection with a flexible laryngoscope showed no other unusual appearance at the larynx with clear vocal cords and symmetrical movement.

Fine needle aspiration (FNA), computed tomography (CT) and magnetic resonance imaging (MRI) were taken to validate malignancy. CT and MRI represented a heterogeneous enhancing mass on the left submandibular gland of $8 \times 4.3 \times$ $8.7 \mathrm{~cm}$ in size with irregular margins extended to the left parapharyngeal space, left mouth floor, left parotid gland, and downward to the left thyroid level (Fig. 1). Atypical cells
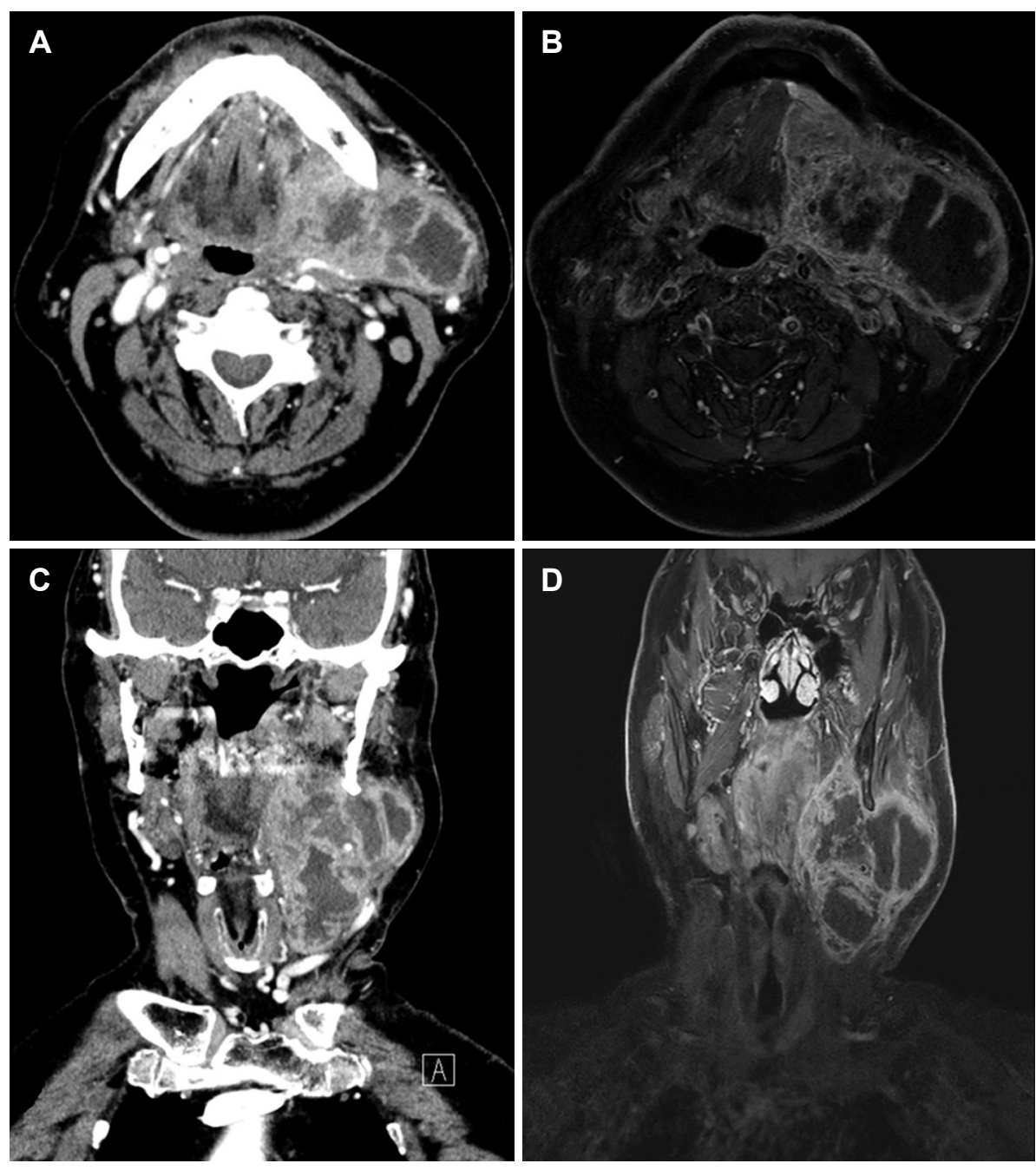

Fig. 1. Pre-operative CT scan axial view $(A)$ showing a heterogenous enhancing mass at the left submandibular gland. MRI on T1-weighted image $(B)$ revealed extension of the mass to the left parapharyngeal space, left mouth floor, left parotid glandwith necrotic portion. The mass extended downwards to the thyroid level in the pre-operative CT scan (C) and MRI T1-weighted image (D) cor-onal view. CT: computed tomography, MRI: magnetic resonance imaging. 
in the FNA was observed in the main mass and contralateral level II lymph node, but it was impossible to eliminate all the possibilities of malignancy. No distant metastasis was detected in the positron emission tomography-CT (PET-CT) test. We decided to perform surgery based on the clinical and radiologic findings.

Under general anesthesia, wide excision of the left submandibular gland tumor, left superficial parotidectomy, left modi-
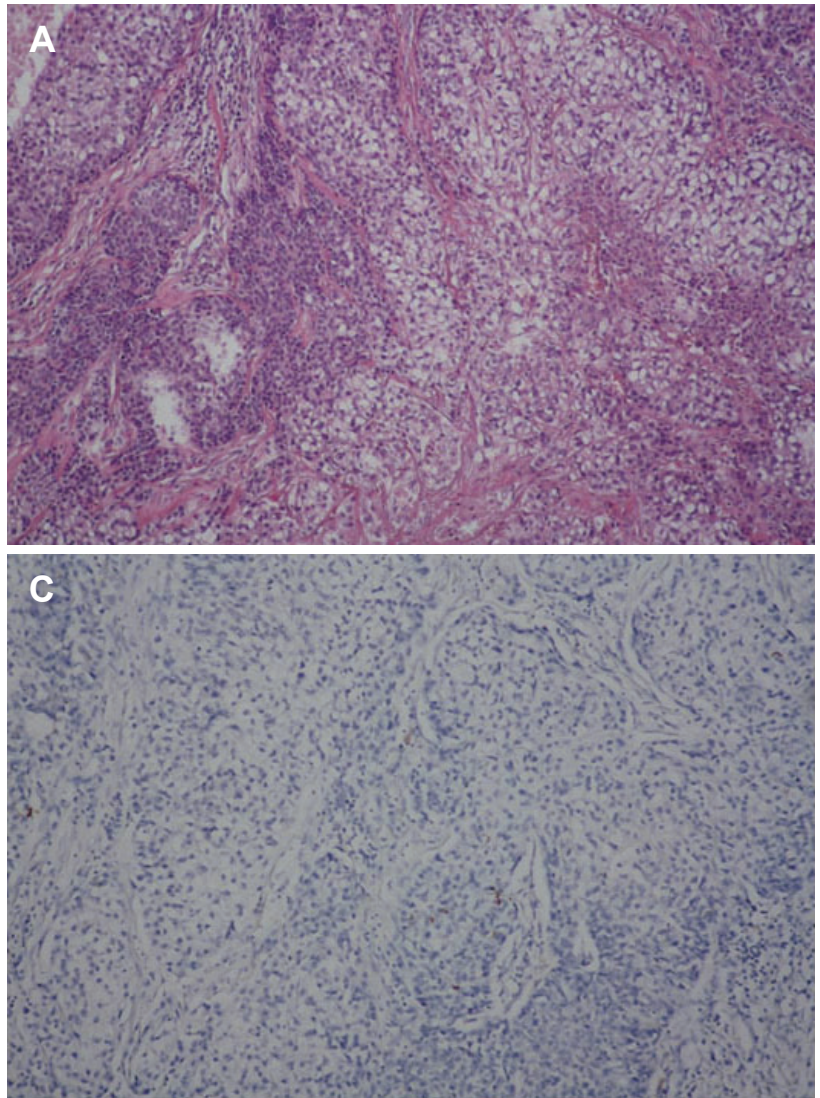

fied radical neck dissection level I, II, III, IV, V, and right selective neck dissection level II, III were performed. No unusual bleeding or other complications was encountered during the surgery. Intraoperative frozen biopsy appeared as malignancy and the resected margins were clear.

Pathologic examinations of the specimen revealed sebaceous carcinoma of low grade differentiation. Immunohistochemical analysis showed negative for S-100 protein and
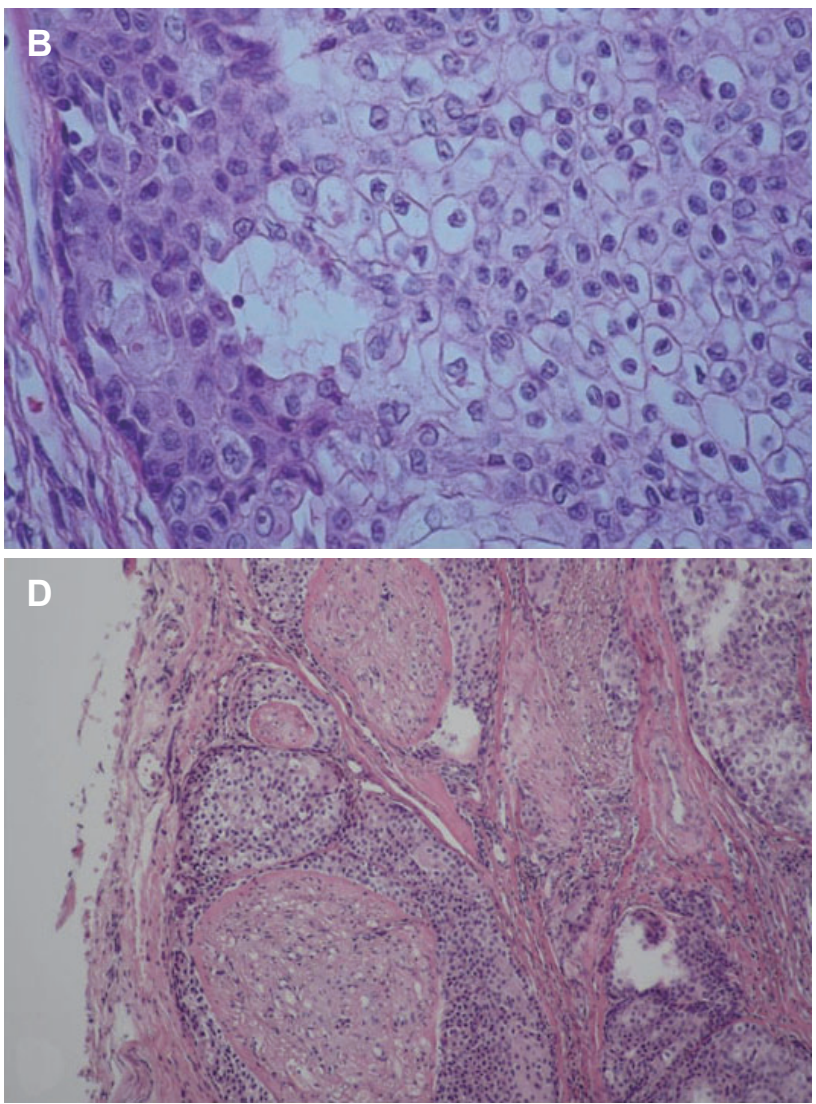

Fig. 2. Histopathologic findings. Cells with clear cytoplasm are observed with basaloid cells (H\&E) (A). A mixture of low-differentiated sebaceous cells with atypical basaloid cells (H\&E) (B). Negative staining for S-100 (C). Perineural infiltration around the nerve bundle (D) (magnification, $\times 100$ in A, C, D, and $\times 400$ in B).

Fig. 3. Pre-operative PET-CT of the left submandibular gland without distant metastasis (A). Post-operative PET-CT 4 months after operation suggesting recurrence of the left submandibular mass. FDG uptake in the right prevertebral area at the L4 level, right pedicle of L2 and a 1.8 $\mathrm{cm}$ sized nodule in the right lower lung (B). PET-CT: positron emission tomography-CT, FDG: fluorodeoxyglucose.

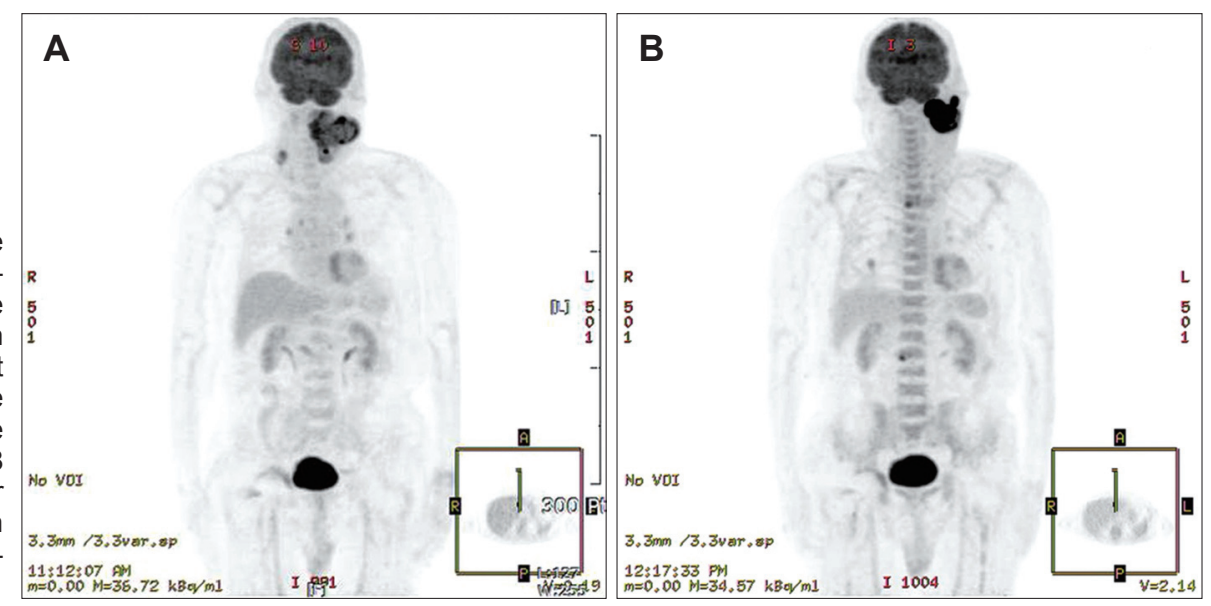


positive for P63. Extraparenchymal extension, perineural infiltration, and necrotic change were observed, however, no lymphovascular invasion was identified (Fig. 2). 2 out of 35 resected lymph nodes were diagnosed as metastatic sebaceous carcinoma of a size of 1.7 and $2 \mathrm{~cm}$. The final staging was stage4a (T4aN2cM0). Adjunctive radiation therapy was recommended but considering the patient's age and medical condition, both the patient and his family refused to take corresponding therapy.

After 5 months from the operation a newly appearing mass was noticed at the left post auricular area. Follow up CT and PET-CT suggested recurrence of the tumor at the left parotid and parapharyngeal area. Fluorodeoxyglucose uptake in the PET-CT suggested of bone and distant metastasis (Fig. 3). Adjuvant radiation therapy was again refused.

\section{Discussion}

Malignant sebaceous tumors of the salivary glands are highly rare, taking up less than $0.2 \%$ of all major salivary gland tumors. ${ }^{9)}$ The parotid glands are the most problematic sites, but malignancy has also been identified at the submandibular gland, oral cavity, sublingual gland, vallecula, epiglottis, and hypopharynx. ${ }^{4,8,9)}$

The range of age affected patients between 17 and 93 with a peak incidence in the third decade and seventh to eighth decades of life. ${ }^{2,89}$ The gender ratio was equally the same 1:1; between male and female. ${ }^{2,8,9)}$

Patients experience pain on their mass of a size ranging from 0.6 to $9.5 \mathrm{~cm}$ as the most common symptom with distinct degree of facial palsy, usually as a later event. ${ }^{2,9,10)}$ In our case, the patient did not show any other symptoms except for swelling in the early years which led to postpone diagnosis and treatment.

Various examinations such as ultrasonography, CT, MRI, PET-CT have been used to assist diagnosis. ${ }^{10)}$ As several tumors arise at the salivary glands, imaging examinations and physical examinations alone are not sufficient enough to draw conclusion and it is known that FNA is not suitable to evaluate the exact history in malignant salivary tumors. ${ }^{1)}$ Because such limits, recent studies emphasize the role on core needle biopsy as a method on diagnosing salivary gland cancer due to a superior accuracy compared to the FNA. ${ }^{11)}$ However in our case the necrotic portion of the mass was large with severe adhesion of the tissues surrounding the mass which concerned us on the complications of core needle biopsy such as facial nerve injury and cancer seeding. Also, clinical and radiological results and atypical cells in the FNA were enough to consider surgery as the primary treatment and intraoperative biopsy was a more reasonable option to reach to the final diagnosis.

Salivary gland tumors are uncommon and its histologic features are so diverse that they make the diagnosis even more difficult. In this respect, hematoxylin-eosin staining is still the gold standard method for diagnosis and immunohistochemistry increases the accuracy. ${ }^{12)}$ Identification of sebocytes is the key portion for the diagnosis of sebaceous tumors. S-100 protein is highly sensitive markers for neoplastic myoepithelial cells and P63 positive for myoepithelial cells and basal cells. ${ }^{12)}$ Considering that perineural invasion has been observed more than $20 \%$ of all tumors, vascular invasion turns out to be extremely rare. ${ }^{9)}$ In our case, sebocytes with atypical basaloid cells were observed in the hematoxylin-eosin staining with negative reaction to S-100 proteins and positive to P63. Perineural invasion was identified but no vascular invasion was found.

The choice of treatment has not been established due to tumor's rarity and its lack of data. ${ }^{1,2,8,9)}$ However, the recommended treatment is based on surgery with wide surgical excision for low grade differentiations. Adjuvant radiotherapy is recommended for high differentiations or positive margins. ${ }^{1,2,8,9)}$ Elective neck dissection should be considered in the case that cancer appears with marked cytologic atypia or when facial nerve involvement is observable. ${ }^{8)}$ In our case, pathologic results was of a low grade differentiation, however, perineural infiltration and contralateral lymph node metastasis were observed as the reason for recommendation of adjuvant radiotherapy.

Chemotherapy is not a recognized standard treatment in the malignant salivary gland tumors. No improvement is shown in the survival outcomes between postoperative chemoradiotherapy and radiotherapy alone in patients who have undergone resection of high risk salivary gland carcinoma. ${ }^{13)}$ The additional chemotherapy is not fully supportive and only case report on systemic treatment with 5-fluorouracil and cisplatin combination chemotherapy is reported with few data on the reaction rate, overall survival rate and toxicity of such treatment. $^{14)}$

Researches of neo-adjuvant chemotherapy of the salivary gland cancer especially of the sebaceous carcinoma were not found. Although there are some reports on the benefit in improvement of the prognosis and possibility of applying 
conservative treatment in advanced stage or metastatic carcinomas of the salivary glands, subsequent concomitant chemotherapy plus radiotherapy is needed and no improvement on the survival appeared. ${ }^{15)}$ In this case, surgery was planned in consideration of surgical resectability and patient age after consultation with the hemato-oncology and radiation oncology department. The surgical margins were negative at the time of surgery but recurred within a relatively short period of time, hence, adjuvant chemoradiotherapy or radiotherapy must be necessary in advanced cases.

In this case recurrence occurred in the parotid area. In the presence of facial nerve involvement, since perineural invasion is a characteristic of sebaceous carcinoma, it is necessary to fully consult with the patient before the operation about any possibilities of total parotidectomy, frozen biopsy of the facial nerve, and sacrification and reconstruction of it.

The overall survival rate for sebaceous carcinoma on the salivary glands is about $62 \%$ which is lower than that of similar cancer arising in the skin and orbit, which is $84.5 \%$. ${ }^{9)} \mathrm{Se}$ baceous carcinoma could possibly recur, whereas metastasis is rare. Seven out of 19 cases (36.8\%) of parotid gland sebaceous carcinoma were reported with local recurrence with or without lymph node metastasis and at least 6 cases of metastasis have been reported. ${ }^{1,8,9)}$

In conclusion, sebaceous carcinoma of the submandibular glands is a rare, slow growing tumor with aggressive features such as distant metastasis occurring in higher stages where adjuvant radiotherapy must be considered. Careful and frequent follow-up must be done to investigate any early recurrence, especially in the high-risk cases such as poor differentiation, lymphovascular infiltration, local invasion or positive margins.

\section{REFERENCES}

1) Takada $Y$, Kawamoto K, Baba S, Takada T, Inoue T, Tomoda $K$.
Sebaceous carcinoma of the parotid gland: a case report. Case Rep Oncol 2015;8(1):106-12.

2) Mighell AJ, Stassen LF, Soames JV. Sebaceous carcinoma of the parotid gland. Dentomaxillofac Radiol 1996;25(1):51-3.

3) Gnepp DR, Brannon R. Sebaceous neoplasms of salivary gland origin. Report of 21 cases. Cancer 1984;53(10):2155-70.

4) Ohara N, Taguchi K, Yamamoto M, Nagano T, Akagi T. Sebaceous carcinoma of the submandibular gland with high-grade malignancy: report of a case. Pathol Int 1998;48(4):287-91.

5) Esnal Leal F, García-Rostan y Pérez GM, Garatea Crelgo J, Gorriarán Terreros M, Arzoz Sainz de Murieta E. [Sebaceous carcinoma of salivary gland. Report of two cases of infrequent location]. An Otorrinolaringol Ibero Am 1997;24(4):401-13.

6) Diedhiou A, Cazals-Hatem D, Rondini E, Sterkers O, Degott C, Wassef M. [Sebaceous carcinoma of the submandibular gland: a case report]. Ann Pathol 2001;21(4):348-51.

7) Takata T, Ogawa I, Nikai H. Sebaceous carcinoma of the parotid gland. An immunohistochemical and ultrastructural study. Virchows Arch A Pathol Anat Histopathol 1989;414(5):459-64.

8) Marnouche el A, Maghous A, Kadiri S, Berhili S, Touil A, Kettani F, et al. Sebaceous carcinoma of the parotid gland: a case report and review of the literature. J Med Case Rep 2016;10:174.

9) Gnepp DR. My journey into the world of salivary gland sebaceous neoplasms. Head Neck Pathol 2012;6(1):101-10.

10) To VS, Chan JY, Tsang RK, Wei WI. Review of salivary gland neoplasms. ISRN Otolaryngol 2012;2012:872982.

11) Douville NJ, Bradford CR. Comparison of ultrasound-guided core biopsy versus fine-needle aspiration biopsy in the evaluation of salivary gland lesions. Head Neck 2013;35(11):1657-61.

12) Nagao T, Sato E, Inoue R, Oshiro H, H Takahashi R, Nagai T, et al. Immunohistochemical analysis of salivary gland tumors: application for surgical pathology practice. Acta Histochem Cytochem 2012;45 (5):269-82

13) Amini A, Waxweiler TV, Brower JV, Jones BL, McDermott JD, Raben $\mathrm{D}$, et al. Association of adjuvant chemoradiotherapy vs radiotherapy alone with survival in patients with resected major salivary gland carcinoma: data from the National Cancer Data Base. JAMA Otolaryngol Head Neck Surg 2016;142(11):1100-10.

14) Orcurto A, Gay BE, Sozzi WJ, Gilliet M, Leyvraz S. Long-term remission of an aggressive sebaceous carcinoma following chemotherapy. Case Rep Dermatol 2014;6(1):80-4.

15) Cortesina G, Airoldi M, Palonta F. Current role of chemotherapy in exclusive and integrated treatment of malignant tumours of salivary glands. Acta Otorhinolaryngol Ital 2005;25(3):179-81. 